\title{
An alternative kernel-based method for estimating copepod growth rates from multimodal biomass distributions in artificial cohort experiments
}

\author{
Kuan-Yu Lin ${ }^{1}$, Akash R Sastri ${ }^{2}$ and Chih-hao Hsieh ${ }^{1,3^{*}}$
}

\begin{abstract}
Background: Elucidating copepod growth and production rates is important in understanding the trophic role of copepods in marine systems. One of the most commonly used field methods for measuring copepod growth rates is the artificial cohort method.

Results: An 'artificial cohort' is established by limiting the incubated animals to relatively narrow size ranges or developmental stages. Thus, one can measure the change in body weight (BW; attributed to body growth) between the start and conclusion of incubation by following the shift in the biomass distribution. The critical issue here is determining how to describe the biomass distribution before and after incubation in a mathematically concise manner. Traditionally, the mean value of the biomass distribution is used as the representative BW, which assumes that the biomass distribution follows a unimodal distribution. However, in practice, the complex composition of copepod communities can commonly yield observations of multimodal distributions. To overcome this difficulty, we suggest that the representative BW of copepod communities be estimated by multiple-peak consideration instead of using the average value. Specifically, we used a kernel-based approach to determine peak values; as such, only BW values associated with a high frequency were used to determine the representative BW.

Conclusions: Through a comparison of different methods, we show that the multiple-peak consideration yielded a higher proportion of realistic growth rate values. In addition, we noted that growth rates estimated with the multiple-peak method were more closely aligned with predictions based on the metabolic theory of ecology.
\end{abstract}

Keywords: Copepod community; Artificial cohort method; Metabolic theory of ecology; Multimodal distribution

\section{Background}

Copepods represent the main component of the marine mesozooplankton, contributing about $80 \%$ to the total abundance (Verity and Smetacek 1996). This group also plays important roles in linking primary production to higher trophic levels in pelagic food webs (Cushing 1989). Importantly, small species (as well as early developmental stages of larger species) represent a key component in marine ecosystems due to their high abundance, wide

\footnotetext{
* Correspondence: chsieh@ntu.edu.tw

${ }^{1}$ Institute of Oceanography, National Taiwan University, 1 Roosevelt Road, Sec. 4, Taipei 106, Taiwan

${ }^{3}$ Institute of Ecology and Evolutionary Biology, National Taiwan University, 1 Roosevelt Road, Sec. 4, Taipei 106, Taiwan

Full list of author information is available at the end of the article
}

distribution (Turner 2004), high weight-specific ingestion rate (Levinsen et al. 2000), and year-round presence and reproduction (Lischka and Hagen 2005; Madsen et al. 2008). Therefore, empirical studies of specific properties of these small copepods such as variations in productivity may help clarify their contributions to marine ecosystem functioning.

Estimates of growth rates are one of the most important processes (Kiørboe 1997) used to investigate the trophodynamics of copepods in marine ecosystems. Several methods were developed to measure copepod growth rates (see Runge and Roff (2000) for a review). One of the best studied and applied field techniques is the artificial cohort (AC) method, which was developed 
by Kimmerer and McKinnon (1987). In practice, the approach relies on the creation and incubation of ACs consisting of selected developmental stages or size fractions (e.g., McKinnon and Duggan 2003). The AC method is often applied in empirical studies (e.g., Hopcroft et al. 1998; McKinnon and Duggan 2003; Kobari et al. 2007) because it relies on fewer assumptions than other approaches (e.g., the molt rate method; Peterson et al. 1991; Hirst et al. 2005). Basically, one only has to assume exponential growth and no artificial interference during incubation; also, the estimation is relatively free from the bias of mortality (Kimmerer et al. 2007).

The key issue in the AC method as discussed here is the ability to obtain a correct biomass distribution of the AC. Under ideal conditions, the AC method should create cohorts with a unimodal distribution of biomass, and thus, the average value can be used as a representative body weight (BW) value for the 'cohort.' However, there are several technical issues in field sampling which can make estimates based on average values inappropriate. For example, most studies only analyzed cohorts of a single developmental stage (nauplius or copepodite); as such, those copepods undergoing a stage transition (e.g., NVI to CI or CV to adult) will be missed in the biomass distribution at the end of incubation (Calbet et al. 2000; Kimmerer et al. 2007). The disappearance of these individuals in transitional stages after incubation can lead to underestimates of growth rates. More importantly, the biomass distribution of copepod assemblages may consist of multiple peaks, despite efforts to create a single peak by the AC technique. This often occurs when the AC method is applied to estimate community growth rates because different species within the community may have different biomass distributions and growth rates. Such an issue is particularly problematic in subtropical and tropical environments where copepod communities are complex and diverse (e.g., Hsieh et al. 2005; Tseng et al. 2008). Furthermore, contamination from imperfect sieving or clogging by algae (Kimmerer et al. 2007; Kobari et al. 2010) can further exacerbate this situation, especially in tropical and subtropical environments where surface waters are often overgrown by microalgae (McKinnon 1996). Under such circumstances, growth rates calculated with simple average biomass values may be biased by the frequency difference between assemblages before and after incubation. For example, if an assemblage before incubation has two peaks and the larger peak is associated with a high BW (i.e., it is highly skewed) and the corresponding assemblage after incubation also has two peaks but the larger peak is now associated with a low BW (as illustrated in Figure 1), the estimated growth rate will be underestimated (and vice versa). One may argue that a way to resolve this issue is to incubate one species at a
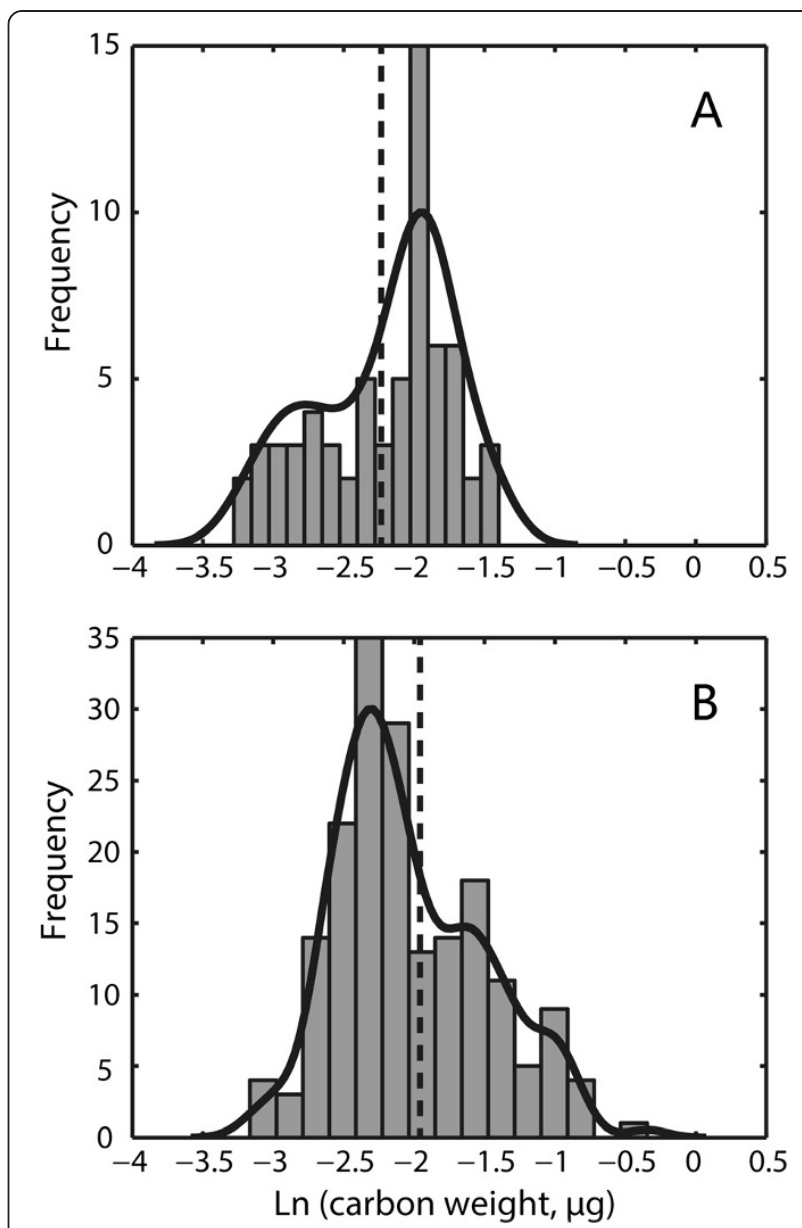

Figure 1 Carbon weight frequency, probability density estimate, and average value of $\ln$ (carbon weight) of copepod assemblages. (A) At $T=0$ and (B) at $T=24$. Bars denote carbon weight frequency. The bold line represents the probability density estimate, and the dashed line represents the average value of In (carbon weight). Note that the $y$-axis of the probability density was rescaled for illustration.

time; however, this is not practical in tropical and subtropical environments where copepod diversity is high and sorting live animals to species level at sea is difficult.

To overcome these technical issues in the AC method for growth rate estimates, we developed a new procedure that accounts for multiple BW peaks in AC experiments. Herein, we measured in situ copepod weight-specific growth rates in the East China Sea by employing the AC method. We demonstrate the efficacy of our new technique of multiple-peak consideration by comparing growth rates estimated with different enumeration methods. First, we compare the number of realistic growth values. Second, we investigate whether growth estimates more closely follow predictions arising from the metabolic theory of ecology (MTE) (Brown et al. 2004) which incorporates both the effects of 
temperature $(T)$ and body mass $(M)$ on the weightspecific growth rate $(g)$ :

$$
g \propto \exp \left(-\frac{E}{k T}\right) \times M^{-0.25},
$$

where $E$ is the enzyme-catalyzed activation energy for biochemical reactions of metabolism and $k$ is Boltzmann's constant $(\mathrm{eV} / \mathrm{K})$. Such an evaluation is based on the observation that the MTE has been widely tested empirically in rate measurements (e.g., Gillooly et al. 2001; Lek et al. 2012; Price et al. 2012) and has theoretical support (Brown et al. 2004).

\section{Methods}

\section{Sampling}

All sampling and incubations were carried out aboard the $R / V$ Ocean Researcher I (OR1) and $R / V$ Ocean Researcher II (OR2) from March 2009 to November 2011. We carried out $\mathrm{AC}$ experiments at 31 stations located in the East China Sea and western Pacific area near Taiwan (Figure 2). Environmental data (e.g., depth-specific temperature) were obtained using a Sea-Bird CTD-General Oceanic Rosette (Sea-Bird Electronics, Inc., Bellevue, WA, USA) equipped with 20-L Go-Flo bottles (General Oceanics, Miami, FL,

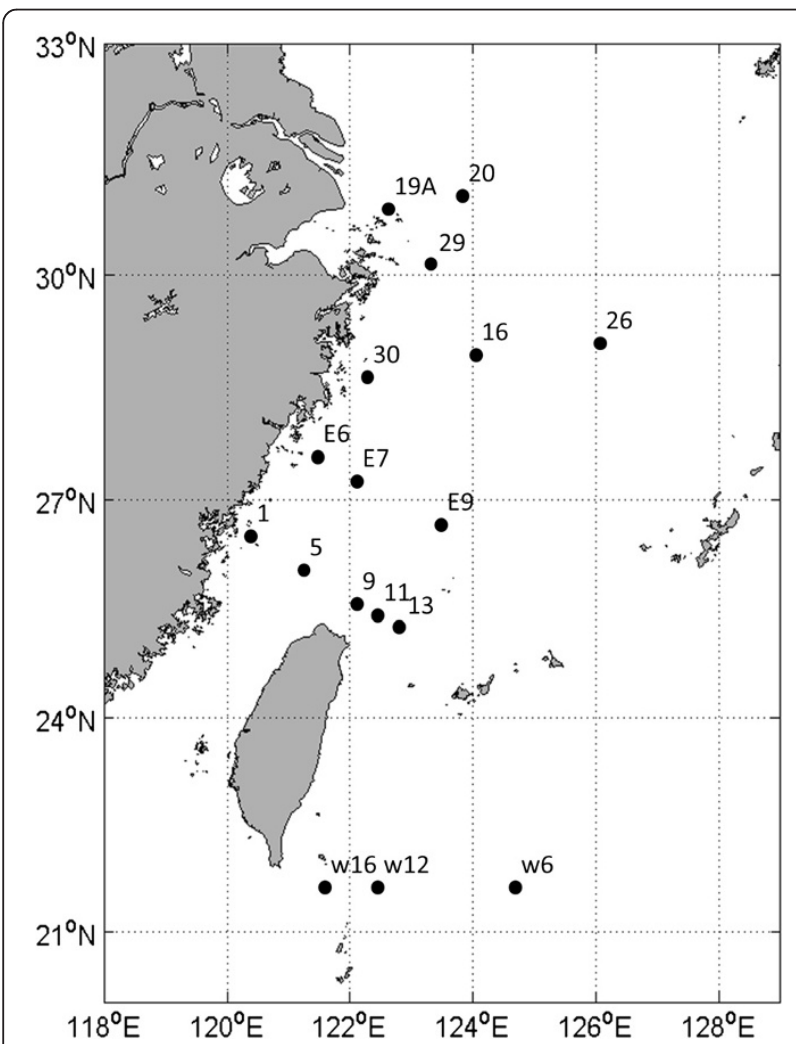

Figure 2 Map showing experimental sites in the East China Sea and Western Pacific Ocean.
USA). Incubation temperatures were measured periodically during incubation or from CTD measurements of surface seawater temperatures if the former measurement was lacking.

\section{AC method}

Copepod weight-specific growth rates were measured using the AC method (Kimmerer and McKinnon 1987). We restricted the body size range of animals by selective filtration in order to create ACs as illustrated in Figure 3. Similar to McKinnon and Duggan (2003), we used 50- to $80-\mu \mathrm{m}$ and $100-$ to $150-\mu \mathrm{m}$ size fractions to respectively isolate nauplii and copepodites. Shipboard incubations for each size fraction were carried out using three replicates in 20-L collapsible polyethylene cubitainers. Incubation seawater (and the food contained therein) was collected from $10 \mathrm{~m}$ using 20-L Go-Flo bottles. This water was screened through a $50-\mu \mathrm{m}$ mesh (in order to exclude mesozooplankton), and the cubitainers were filled to approximately $90 \%$ capacity. Seawater accompanying the size-fractionated zooplankton made up the remaining volume of each 20-L cubitainer.

Live zooplankton (mainly copepods) were collected using two separate Norpac zooplankton nets (50- and $100-\mu \mathrm{m}$ mesh, respectively, and each with a ring diameter of $45 \mathrm{~cm}$; General Oceanics, Miami, FL, USA). At each station, nets were set to $10 \mathrm{~m}$ in depth and allowed to drift with the ship for 5 to $10 \mathrm{~min}$. The contents of each net were carefully resuspended in buckets filled with prescreened incubation seawater. After gentle mixing, the contents of the $50-\mu \mathrm{m}$ net were reverse filtered through an $80-\mu \mathrm{m}$ mesh and siphoned (approximately $2 \mathrm{~L}$ ) into cubitainers for the 50- to $80-\mu \mathrm{m}$ AC incubations. Another subsample from the $80-\mu \mathrm{m}$-mesh reverse filtrate, representing the biomass distribution at the start of the incubation (i.e., time 0), was preserved with $5 \%$ formalin-buffered seawater. The process was repeated using the contents of the $100-\mu \mathrm{m}$-mesh net and reverse filtered with a $150-\mu \mathrm{m}$ mesh to establish a 100 to $150-\mu \mathrm{m}$ AC. All cubitainers were incubated in dark black tanks (of about $200 \mathrm{~L}$ in volume) filled with circulating seawater pumped constantly from the surface during each cruise. Incubation periods were $24 \mathrm{~h}$ for the $50-$ to $80-\mu \mathrm{m}$ size fraction and $48 \mathrm{~h}$ for the $100-$ to $150-\mu \mathrm{m}$ size fraction in order to allow sufficient time for measurable growth to occur. The lengths of the incubation periods were also carefully considered; therefore, we assumed no container effects (e.g., food limitation) for the organisms in the cubitainers during these periods. After incubation, the contents of each cubitainer were concentrated onto a $50-\mu \mathrm{m}$ mesh and preserved with $5 \%$ formalin-buffered seawater.

\section{Classification and growth rate estimation}

Preserved samples were identified and counted using a dissecting microscope, and images of $8 \times 10$ magnification 


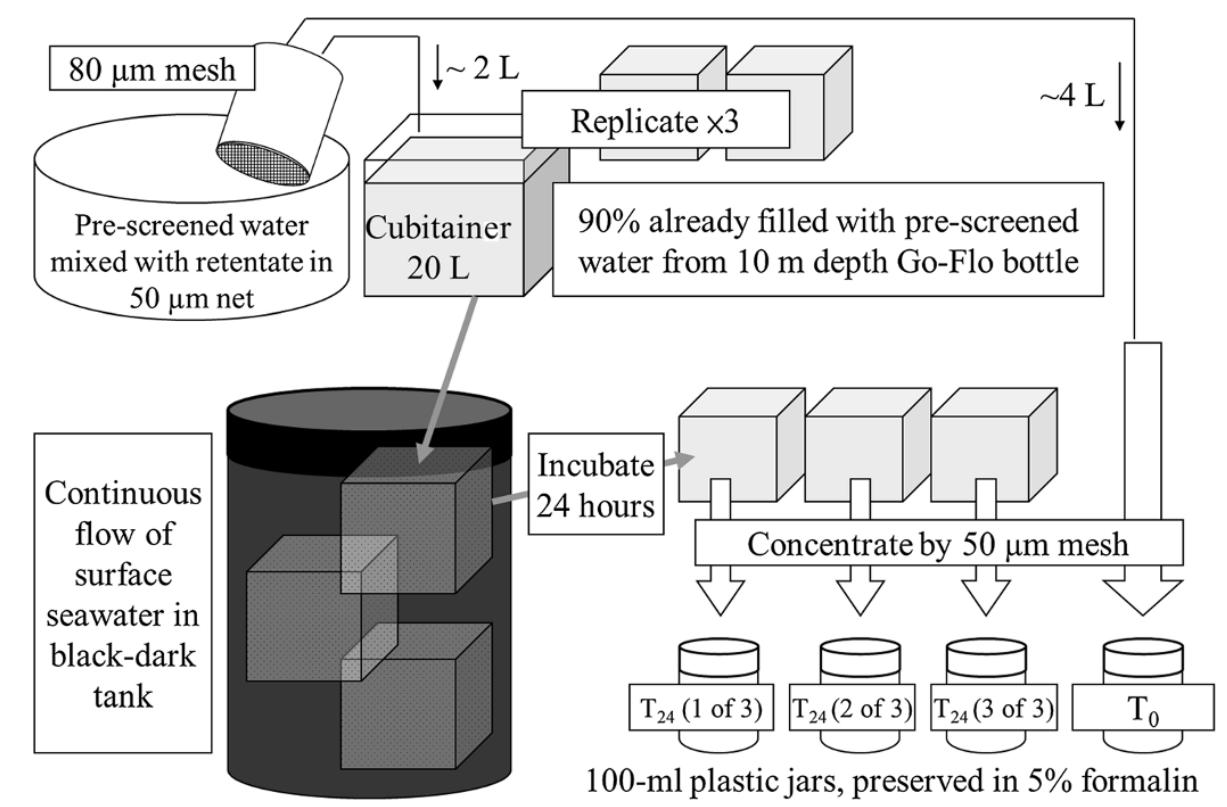

Figure 3 Schematic diagram illustrating incubation experiments for the $\mathbf{5 0}$ - to $\mathbf{8 0}-\boldsymbol{\mu m}$ size fraction. Animals from the retentate were confined within the size range of 50 to $80 \mu \mathrm{m}$ using the plankton net and mesh. Three replicates were carried out, incubated in tanks for $24 \mathrm{~h}$, and preserved in $5 \%$ formalin $\left(T_{24}\right)$. Additional preservation of $T_{0}$ was also done at the beginning of incubation.

were taken using a CCD camera (Olympus DP71 with the software analySIS LS Starter 2.6; Olympus America Inc., Center Valley, PA, USA) mounted on the microscope. We followed the protocol of McKinnon and Duggan (2003) and limited our analysis to copepod morphotypes rather than individual species (e.g., Kimmerer and McKinnon 1987; Liu and Hopcroft 2006a, b; Kobari et al. 2007). In the $50-$ to $80-\mu \mathrm{m}$ size fraction, our morphotypes were calanoid (Calanoida) and cyclopoid (Cyclopoida) nauplii. We occasionally found harpacticoid (Harpacticoida) nauplii in our incubations, and these animals were measured and enumerated when sufficiently abundant. In the $100-$ to $150-\mu \mathrm{m}$ size fraction, we identified, counted, and measured calanoid, oithonid (Cyclopoida: Oithonidae), harpacticoid, oncaeid (Poecilostomatoida: Oncaeidae), and corycaeid (Poecilostomatoida: Corycaeidae) copepodites in addition to calanoid and cyclopoid nauplii. The taxonomic resolution of identification was determined under consideration of the trade-off with sample sizes. Each morphotype contributed to one growth rate value (if available) from a single incubation.

The prosome length and width of each individual were measured from digital images of the copepods. Body mass metrics for morphotypes of different shapes were calculated according to Svetlichny (1983):

Wet weight $(\mathrm{WW})=K_{c} \times$ Prosomal length $\times$ Width $^{2}$, where $K_{c}$ is a constant ( 0.6 for calanoids and 0.705 for cyclopoids; McKinnon and Duggan 2003) and has an average value of 0.65 for groups where conversion factors were not available. A conversion factor of $0.135 \times 0.42$ was used to convert the wet weight to carbon weight, i.e.,

Dry weight $(\mathrm{DW})=0.135 \times \mathrm{WW}$

(Postel et al. 2000) and

$$
\text { Carbon weight }(W)=0.42 \times \mathrm{DW}
$$

(Beers 1966).

Assuming exponential growth (e.g., Kimmerer et al. 2007), the weight-specific growth rate $(g)$ was calculated as

$$
g=\ln \left(\frac{W_{T}}{W_{0}}\right) / T,
$$

where $W_{0}$ is the carbon weight of copepods at the beginning of incubation, $W_{T}$ is the carbon weight at the end of incubation, and $T$ represents the respective incubation times of 24 and $48 \mathrm{~h}$ for the $50-$ to $80-\mu \mathrm{m}$ and $100-$ to $150-\mu \mathrm{m}$ size fractions.

\section{Calculation and comparison of three estimates of the representative $\mathrm{BW}$}

The representative BW value (in units of carbon) for each copepod assemblage (i.e., $W_{0}$ and $W_{T}$ ) was estimated by three methods for comparison: (1) the average value, (2) mode value, and (3) multiple-peak consideration. Only 
assemblages with $\geq 30$ individual copepods were used here (the average number for each morphotype was approximately 160 and ranged from 30 to 1,182). The first method using the average value was the same as the traditional method for BW estimation: without prior examination of the biomass distribution, the representative $\mathrm{BW}$ value was calculated as the geometric mean value of the BW of all copepod individuals for each assemblage of each morphotype (e.g., $W_{0}=\exp (-2.31)$, the dashed line in Figure $1 \mathrm{~A} ; W_{24}=$ $\exp (-2.02)$, the dashed line in Figure 1B).

The procedures for the methods considering the mode value and multiple peaks were as follows. In the first step, we calculated the probability density estimate (PDE) using $\ln (\mathrm{BW})$ values of all copepod individuals by a kernel-smoothing technique (see Figure 1 for an illustration) for each assemblage of each morphotype. First, a discrete distribution of biomass was approximated by a continuous distribution based on a normal kernel function (Bowman and Azzalini 1997), with a bandwidth of the kernel-smoothing window of 0.1. Second, local maximal values (peaks) of the PDE were located and singled out. To avoid any bias caused by outliers, we excluded minor peaks that had a probability density (peak height) of $<1 / 3$ the height of the largest peak (e.g., the peaks where BWs were $\exp (-1.00)$ and $\exp (-0.32)$ in Figure 1B were accordingly excluded). Third, unreasonable values (which might arise from contamination, experimental failure, or different growth rates of organisms within cohorts) were removed. The criteria for removal were as follows: for any peak $\mathrm{BW}$ at $T=0$ that was higher than all peak BWs at $T=24 / 48$, that peak at $T=0$ was eliminated. Similarly, for any peak BW at $T=24 / 48$ that was lower than all peak BWs at $T=0$, that peak at $T=24 / 48$ was also eliminated. If there was no remaining peak at $T=0$ or all $T=24 / 48$, the growth rate was not calculated. Following this criterion, only one or two (rarely three) peaks ever remained for each assemblage. The elimination of unreasonable values based on our criteria also meant that there were fewer values remaining for calculation based on the mode value and multiple-peak consideration than with the average value approach. Unbalanced numbers of peaks between $T=0$ and $T=24 / 48$ were permitted in growth rate calculations because copepod growth patterns can vary as a consequence of the complex composition of a diverse assemblage (as described above).

For the method based on the mode value, only the $\mathrm{BW}$ value associated with the largest peak was chosen to represent the $\mathrm{BW}$ value for the assemblage (e.g., $W_{0}=$ $\exp (-1.98)$ in Figure 1A; $W_{24}=\exp (-2.41)$ in Figure 1B). The mode value method is a preliminary consideration of peaks, and it can be regarded as an intermediate method between the traditional average value method and multiple-peak consideration. For the multiple-peak consideration, the average value (or single value if only one peak was identified) of the peak BWs was the representative $\mathrm{BW}$ value for the assemblage (e.g., $W_{0}=\exp$ $(-2.44)$ according to the two peaks of -2.90 and -1.98 in Figure 1A). Note that we have only one $T=0$ sample and three $T=24 / 48$ replicates. Thus, three pairs of $\left[W_{0}-W_{T}\right]$ for one incubation for each morphotype were respectively calculated by each enumeration method. Each pair of $\left[W_{0^{-}}\right.$ $W_{T}$ ] was used to calculate growth rate values by Equation 5 . Finally, the growth rate estimates represent the average of the three values from the replicates.

To help illustrate differences in performance among the three methods, it is useful to show an example. According to the example shown in Figure 1, the weight-specific growth rate calculated by the average value method was $-2.02-(-2.31)=0.29$. However, an obvious underestimation can be observed in Figure 1 due to the negative-skewed biomass distribution in $T=0$ and the positive-skewed form in $T=24$. This problem was resolved by the multiple-peak consideration. Two peaks were extracted in both $T=0$ (-2.90 and -1.98 , average -2.44$)$ and $T=24(-2.41$ and -1.61 , average -2.01$)$ assemblages, and a more appropriate estimate of the weightspecific growth rate was calculated as $-2.01-(-2.44)=$ 0.37 . The mode value method yielded an unrealistic negative growth rate estimate of -0.43 .

\section{Testing the efficacy of each method for growth rate estimation}

In order to test the efficacy of the three methods, we performed two types of comparison. First, we simply compared the calculated growth rate values among the three methods (the pooled data included all morphotypes as representative). Specifically, the number of realistic growth rate values (weight-specific growth rate from 0 to 2) was compared. If the proportion of realistic growth rate estimates was higher for any given method, then that method was considered a better approach. Second, we fitted growth rate estimates to the relationship predicted by the MTE (Brown et al. 2004). We evaluated the goodness of fit and whether the estimated coefficient of temperature and body mass followed the prediction of the MTE for each of the three methods. The generalized linear model (GLM) is described as

$$
\ln (g)=a_{0}+\frac{a_{1}}{T}+a_{2} \ln (M)
$$

where the coefficient $a_{0}$ is the intercept, $a_{1}$ is the factor of activation energy associated with temperature, and $a_{2}$ is the allometric coefficient for body mass. Here, the body mass $(M)$ was measured as the carbon BW at the beginning of incubation (i.e., $W_{0}$, which could differ due to different methods in use) for each copepod assemblage.

In addition to the analysis of pooled data, we also performed the same GLM analysis on selected abundant 
Table 1 Number of total and realistic growth rate estimates for different methods

\begin{tabular}{lccc}
\hline & $\begin{array}{c}\text { Number of total } \\
\text { growth rate } \\
\text { estimates }(\boldsymbol{N})\end{array}$ & $\begin{array}{c}\text { Number of } \\
\text { realistic growth } \\
\text { rate estimates } \\
(\boldsymbol{n})\end{array}$ & $\begin{array}{c}\text { Percentage of } \\
\text { realistic growth rate } \\
\text { estimates }(\boldsymbol{n} / \boldsymbol{N}, \boldsymbol{\%})\end{array}$ \\
\hline $\begin{array}{l}\text { Method } \\
1\end{array}$ & 196 & 168 & 86 \\
$\begin{array}{l}\text { Method } \\
2\end{array}$ & 181 & 181 & 100 \\
$\begin{array}{l}\text { Method } \\
3\end{array}$ & 189 & 189 & 100 \\
\hline
\end{tabular}

The methods are as follows: (1) average value, (2) mode value, and (3) multiple-peak consideration. Due to elimination of unreasonable values using the criteria for removal (see "Methods") before the growth rate estimation, there were fewer than 196 values for the methods with the mode value (method 2) and multiple-peak consideration (method 3).

morphotypes, 'calanoid' and 'cyclopoid' (which combined oithonids, oncaeids, and corycaeids), in the 100- to $150-\mu \mathrm{m}$ size fraction as representative groups. Moreover, a 10-fold cross-validation (Cudeck and Browne 1983) was performed to compare the GLM results. That is, we randomly divided the dataset into 10 subsamples and used 9 subsamples to construct the GLM model and the remaining subsample to evaluate the model performance (based on a prediction error). The same procedure was repeated 10 times to exhaust the 10 combinations, and the average error was calculated. A small prediction error was considered good performance.

\section{Statistical analysis}

The growth rate values calculated with each of the three calculation methods were compared using a one-way analysis of variance (ANOVA). We used a GLM (Equation 6) to evaluate (1) the goodness of fit of our three types of growth rate estimates to MTE predictions and (2) the correspondence of our estimated coefficients for temperature and body size to the MTE coefficients. All of the statistical analyses used in this study were carried out with MATLAB (The Mattworks, Inc., Concord, MA, USA).

\section{Results}

Efficacy test 1: the number of realistic growth estimates In total, 196 growth rate values (including all morphotypes in both size fractions) were obtained with the average value method (Table 1). However, due to elimination of unreasonable values using the criteria for removal (see "Methods" above), there were only 181 and 189 values for the methods of mode value and multiple-peak consideration, respectively (Table 1 ). No significant differences among the three methods in weight-specific growth rates were found (one-way ANOVA, $F=0.51, p=0.60$ ). When testing the efficacy of each approach using the number of realistic growth rate values, we found that there were 168, 181 , and 189 growth values within the range of 0 and 2 for enumeration by the average value, mode value, and multiple-peak consideration, respectively (Table 1 ). The traditional average value method yielded the lowest percentage of realistic growth rate estimates (Table 1).

\section{Efficacy test 2: regression with temperature and body mass}

Weight-specific growth rates were positively related to temperature and negatively related to body mass, regardless of which method was applied (Table 2). The coefficient of determination $\left(r^{2}\right)$ was lowest when the average value method was applied. The range of temperature coefficients overlapped with values predicted by the MTE ( $E=0.6$ to $0.7 \mathrm{eV}$; Gillooly et al. 2001) for all three methods. However, the coefficient for body mass did not approach the predicted value $(-0.25)$ for rates estimated by any of the three methods (Table 2). Only the method of multiple-peak consideration yielded a less negative body size coefficient estimation, which more closely approached the predicted MTE value of -0.25 (Table 2). In addition, the method of multiple-peak consideration yielded the lowest mean squared error (MSE) for the 10-fold cross-validation (Table 2). An observation of this type was also qualitatively similar when we respectively analyzed data for the calanoid (Table 3) and cyclopoid (Table 4) subgroups.

Table 2 Regression coefficients of temperature and body mass in relation to the weight-specific growth rate

\begin{tabular}{|c|c|c|c|c|c|c|c|}
\hline & $\begin{array}{c}a_{0} \\
\text { (growth constant) }\end{array}$ & $\begin{array}{c}E \\
\text { (activation energy) }\end{array}$ & $\begin{array}{c}a_{2} \\
\text { (size coefficient) }\end{array}$ & $r^{2}$ & $\begin{array}{c}p \\
\text { value }\end{array}$ & $n$ & $\begin{array}{c}\text { MSE of } \\
\text { cross-validation }\end{array}$ \\
\hline Method 1 & $13.48( \pm 7.99)$ & $0.44( \pm 0.21)$ & $-0.60( \pm 0.08)$ & 0.20 & $<0.01$ & 168 & 0.71 \\
\hline Method 2 & $11.21( \pm 8.06)$ & $0.39( \pm 0.21)$ & $-0.71( \pm 0.09)$ & 0.28 & $<0.01$ & 181 & 0.72 \\
\hline Method 3 & $14.13( \pm 6.34)$ & $0.44( \pm 0.16)$ & $-0.49( \pm 0.06)$ & 0.26 & $<0.01$ & 189 & 0.43 \\
\hline Expected value & & 0.6 to 0.7 & -0.25 & & & & \\
\hline
\end{tabular}

The regression coefficients were calculated according to the function $\ln g=a_{0}+a_{1} T+\mathrm{a}$. The methods are as follows: (1) average value, (2) mode value, and (3) multiple-peak consideration. Values in parentheses are bootstrap estimates of the standard error of coefficients. $g$, weight-specific growth rate (1/day); $M$, body mass $(\mu \mathrm{g}) ; T$, temperature $(\mathrm{K}) ; E$, activation energy $(\mathrm{eV}), E=-a_{1} \times k ; k$, Boltzmann's constant (eV/K); $n$, sample size; MSE of cross-validation, mean squared error calculated from the 10 -fold cross-validation analysis. 
Table 3 Regression coefficients of temperature and body mass in relation to weight-specific growth rate of calanoid morphotype

\begin{tabular}{|c|c|c|c|c|c|c|c|}
\hline & $\begin{array}{c}a_{0} \\
\text { (growth constant) }\end{array}$ & $\begin{array}{c}E \\
\text { (activation energy) }\end{array}$ & $\begin{array}{c}a_{2} \\
\text { (size coefficient) }\end{array}$ & $r^{2}$ & $\begin{array}{c}p \\
\text { value }\end{array}$ & $n$ & $\begin{array}{c}\text { MSE of } \\
\text { cross-validation }\end{array}$ \\
\hline Method 1 & $3.11( \pm 11.26)$ & $0.16( \pm 0.29)$ & $-0.55( \pm 0.17)$ & 0.12 & 0.01 & 72 & 1.97 \\
\hline Method 2 & $-2.52( \pm 9.76)$ & $-0.00( \pm 0.25)$ & $-0.33( \pm 0.13)$ & 0.09 & 0.04 & 74 & 1.13 \\
\hline Method 3 & $15.73( \pm 11.82)$ & $0.46( \pm 0.31)$ & $-0.31( \pm 0.12)$ & 0.14 & $<0.01$ & 76 & 0.80 \\
\hline Expected value & & 0.6 to 0.7 & -0.25 & & & & \\
\hline
\end{tabular}

The regression coefficients were calculated according to the function Ing $=a_{0}+a_{1} T+a$. The methods are as follows: (1) average value, (2) mode value, and (3) multiple-peak consideration. Values in parentheses are bootstrap estimates of standard error of coefficients. $g$, weight-specific growth rate (1/day); $M$, body mass $(\mu \mathrm{g})$; $T$, temperature $(\mathrm{K}) ; E$, activation energy $(\mathrm{eV}), E=-a_{1} \times k ; k$, Boltzmann's constant $(\mathrm{eV} / \mathrm{K})$; $n$, sample size; MSE of cross-validation, mean squared error calculated from the 10 -fold cross-validation analysis.

\section{Discussion}

In the current study, we introduced a new method of representative BW determination by multiple-peak consideration for copepod growth rate estimations. We also compared the new method with the traditional average value method by evaluating the number of realistic growth rate values and their accordance with the MTE, in order to demonstrate the performance of the multiple-peak consideration method.

We found that the method of multiple-peak consideration provided better estimates of growth rate values by yielding a higher proportion of realistic growth values (Table 1). The reason is that enumeration by the multiplepeak consideration was able to resolve problems that the traditional average value method faces. First, multiple-peak consideration avoids underestimating the representative BW when stage transitions occur during incubation. If there were no possible corresponding BW peaks after incubation (i.e., the inevitability of missed individuals after a stage transition), that BW peak before incubation would not be considered in the calculation. Second, samples must be discarded in traditional enumeration if sieving problems (leakage or clogging, e.g., Kobari et al. 2010) lead to contamination; otherwise, an abnormal distribution of biomass will result in a potential bias or higher variability in growth rate values (Kimmerer et al. 2007). By applying the multiple-peak consideration, the contamination effect was minimized because BW values of a low frequency are not considered when calculating the growth rate. Third, due to the complexity of copepod communities, one apparent cohort may consist of organisms with different growth rates. Enumeration with the multiple-peak consideration can take such a condition into account, and even the occasional unreasonable condition (e.g., negative growth) can also be handled according to the criteria for removal.

We also investigated how well the growth rates estimated with multiple-peak consideration followed predictions using the MTE (Brown et al. 2004) which incorporates the effects of both temperature $(T)$ and body mass $(M)$ on weight-specific growth rates. Results from all three enumeration methods were qualitatively consistent with the prediction of the MTE: growth rates were positively related to temperature and negatively related to body mass (Table 2), which were also consistent with most studies of copepod growth rates (e.g., Campbell et al. 2001; Hirst and Bunker 2003). However, the coefficient of determination $\left(r^{2}\right)$ showed that the two methods that considered mode (peak) values performed better than the traditional method that considered average values. Additionally, in spite of similar estimates of temperature coefficients among the three methods, the multiple-peak consideration method yielded a coefficient of body mass

Table 4 Regression coefficients of temperature and body mass in relation to weight-specific growth rate of cyclopoid morphotype

\begin{tabular}{|c|c|c|c|c|c|c|c|}
\hline & $\begin{array}{c}a_{0} \\
\text { (growth constant) }\end{array}$ & $\begin{array}{c}E \\
\text { (activation energy) }\end{array}$ & $\begin{array}{c}a_{2} \\
\text { (size coefficient) }\end{array}$ & $r^{2}$ & $\begin{array}{c}p \\
\text { value }\end{array}$ & $n$ & $\begin{array}{c}\text { MSE of } \\
\text { cross-validation }\end{array}$ \\
\hline Method 1 & $18.44( \pm 11.15)$ & $0.57( \pm 0.29)$ & $-0.59( \pm 0.12)$ & 0.24 & $<0.01$ & 81 & 1.20 \\
\hline Method 2 & $14.40( \pm 9.58)$ & $0.47( \pm 0.25)$ & $-0.70( \pm 0.10)$ & 0.33 & $<0.01$ & 88 & 0.76 \\
\hline Method 3 & $11.69( \pm 8.68)$ & $0.38( \pm 0.22)$ & $-0.51( \pm 0.08)$ & 0.27 & $<0.01$ & 96 & 0.55 \\
\hline Expected value & & 0.6 to 0.7 & -0.25 & & & & \\
\hline
\end{tabular}

The regression coefficients were calculated according to the function $\ln g=a_{0}+a_{1} T+a$. The methods are as follows: (1) average value, (2) mode value, and ( 3 ) multiple-peak consideration. Values in parentheses are bootstrap estimates of the standard error of coefficients. $g$, weight-specific growth rate (1/day); $M$, body mass $(\mu \mathrm{g}) ; T$, temperature $(\mathrm{K}) ; E$, activation energy $(\mathrm{eV}), E=-\mathrm{a}_{1} \times k ; k$, Boltzmann's constant (eV/K); $n$, sample size; MSE of cross-validation, mean squared error calculated from the 10 -fold cross-validation analysis. The cyclopoid morphotype includes oithonids, oncaeids, and corycaeids in the 100 - to 150 - $\mu \mathrm{m}$ size fraction. 
that was closer to the predicted value of the MTE (Table 2). In order to carefully examine the efficacy of MTE fitting, we also carried out 10-fold cross-validation of the regression between growth rate estimates and variables described by the MTE (i.e., temperature and body mass). The results were qualitatively similar to those of the GLM regression analysis; the MSE was lowest when applying the multiple-peak consideration (0.43, compared to 0.71 and 0.72 for the average value and mode value methods, respectively). The better performance of the multiple-peak consideration was further revealed when we focused only on representative morphotypes (calanoids and cyclopoids): only the method of multiple-peak consideration yielded coefficients of body mass and temperature which overlapped predicted MTE values (Tables 3 and 4). In addition, results from the method of multiple-peak consideration yielded the greatest coefficient of determination $\left(r^{2}\right)$ and the lowest MSE in the analysis that focused on calanoids (Table 3) and cyclopoids (Table 4). However, due to a relatively low number of data points for regression, the coefficient of determination was even lower (compared to results of the pooled data in Table 2). Certainly, a higher consistency with MTE cannot be sufficient proof of a better prediction of growth rate variation. Nevertheless, our new method indeed helped resolve some technical difficulties, such as the multimodal distribution of biomass that cannot be resolved with the traditional method.

In addition to the issue of enumeration methods, we also noted some caveats associated with sampling. Samples from different seasons or locations may be affected by different thermal or food conditions. Food resources available during incubation are indeed crucial for the growth of copepods (Lin et al. 2013), and this was also one of the reasons we chose the incubation time with an intermediate duration (the incubation time can be even longer when conducted in temperate areas). In order to completely understand the dynamics of copepod communities, further examination of seasonality and/or stage/ species-specific information is required. However, there is always a trade-off between time and taxonomic resolution. This is particularly a challenge in tropical and subtropical environments. Nevertheless, this study focused on improving the data analysis of estimating growth rates and demonstrated the applicability of our method.

In this study, we sought to remove unreasonable growth rates from our analyses. However, we note here that some of those unreasonable growth rates may signal biological information. For example, some of them may be related to food-limited growth for large animals or mortality. In addition, an apparent widening of the cohorts during the course of the experiment may occur when applying the $\mathrm{AC}$ experiment as a result of differences in ages within stages in the initial cohort. Depending on the research question, one may want to treat these data separately in order to develop a comprehensive understanding of environmental effects.

\section{Conclusions}

In conclusion, our results suggest that the new method of multiple-peak consideration offers a better estimation of weight-specific growth rates when resolving rates at the community level. The improvement might be most applicable in tropical/subtropical systems where problems with traditional methods can be quite significant. Further application and examination of this new method are encouraged. Through better estimates of copepod community growth rates, we hope to improve our knowledge of the ecology of copepods and further refine our understanding of the functional roles of this group in marine ecosystems.

\section{Competing interests}

The authors declare that they have no competing interests.

\section{Authors' contributions}

KY carried out the sampling, collected data from microscopic work, performed the statistical analysis, and drafted the manuscript. AR participated in the design of the study, sampling, and data collection. $\mathrm{CH}$ conceived the study and participated in its design and coordination. All authors read and approved the final manuscript.

\section{Acknowledgements}

We acknowledge the assistance provided by the crews of the RN ORI and $R /$ V ORII during sampling and Y.C. Lee and W.H. Teng with the experiments. Comments from C.W. Chang, T.S. Chiu, C. García-Comas, T. Miki, and C.t. Shih greatly improved this work. This study was supported by National Taiwan University and the National Science Council (NSC) of Taiwan.

\section{Author details}

${ }^{1}$ Institute of Oceanography, National Taiwan University, 1 Roosevelt Road, Sec. 4, Taipei 106, Taiwan. ${ }^{2}$ Department of Biological Sciences, Université du Québec à Montréal, Montreal H3C 3P8, Canada. ${ }^{3}$ Institute of Ecology and Evolutionary Biology, National Taiwan University, 1 Roosevelt Road, Sec. 4, Taipei 106, Taiwan.

Received: 23 October 2012 Accepted: 4 March 2013

Published: 25 September 2013

\section{References}

Beers JR (1966) Studies on the chemical composition of the major zooplankton groups in the Sargasso Sea off Bermuda. Limnol Oceanogr 11:520-528

Bowman AW, Azzalini A (1997) Applied smoothing techniques for data analysis. Oxford University Press, New York

Brown JH, Gillooly JF, Allen AP, Savage VM, West GB (2004) Toward a metabolic theory of ecology. Ecology 85:1771-1789

Calbet A, Trepat I, Arin L (2000) Naupliar growth versus egg production in the calanoid copepod Centropages typicus. J Plankton Res 22:1393-1402

Campbell RG, Wagner MM, Teegarden GJ, Boudreau CA, Durbin EG (2001) Growth and development rates of the copepod Calanus finmarchicus reared in the laboratory. Mar Ecol Progr Ser 221:161-183

Cudeck R, Browne MW (1983) Cross-validation of covariance structures. Multivar Behav Res 47:147-167

Cushing DH (1989) A difference in structure between ecosystems in strongly stratified waters and in those that are only weakly stratified. J Plankton Res 11:1-13

Gillooly JF, Brown JH, West GB, Savage VM, Charnov EL (2001) Effects of size and temperature on metabolic rate. Science 293:2248-2251

Hirst AG, Bunker AJ (2003) Growth of marine planktonic copepods: global rates and patterns in relation to chlorophyll $a$, temperature, and body weight. Limnol Oceanogr 48:1988-2010 
Hirst AG, Peterson WT, Rothery P (2005) Errors in juvenile copepod growth rate estimates are widespread: problems with the moult rate method. Mar Ecol Progr Ser 296:263-297

Hopcroft RR, Roff JC, Webber MK, Witt JDS (1998) Zooplankton growth rates: the influence of size and resources in tropical marine copepodites. Mar Biol 132:67-77

Hsieh CH, Chen CS, Chiu TS (2005) Composition and abundance of copepods and ichthyoplankton in Taiwan Strait (western North Pacific) are influenced by seasonal monsoons. Mar Freshw Res 56:153-161

Kimmerer WJ, McKinnon AD (1987) Growth, mortality and secondary production of the copepod Acartia tranteri in Westernport Bay. Aust Limnol Oceanogr 32:14-28

Kimmerer WJ, Hirst AG, Hopcroft RR, McKinnon AD (2007) Estimating juvenile copepod growth rates: corrections, inter-comparisons and recommendations. Mar Ecol Progr Ser 336:187-202

Kiørboe T (1997) Population regulation and role of mesozooplankton in shaping marine pelagic food webs. Hydrobiologia 363:13-27

Kobari T, Imamura S, Habano A (2007) Growth rate of predominant copepods in Kagoshima Bay - estimation by artificial cohort method. Mem Fac Fish Kagoshima Univ 56:45-54

Kobari T, Ueda A, Nishibe Y (2010) Development and growth of ontogenetically migrating copepods during the spring phytoplankton bloom in the Oyashio region. Deep-Sea Res II 57:1715-1726

Lek E, Fairclough DV, Hall NG, Hesp SA, Potter IC (2012) Do the maximum sizes, ages and patterns of growth of three reef-dwelling labrid species at two latitudes differ in a manner conforming to the metabolic theory of ecology? J Fish Biol 81:1936-1962

Levinsen H, Turner JT, Nielsen TG, Hansen BW (2000) On the trophic coupling between protists and copepods in Arctic marine ecosystems. Mar Ecol Progr Ser 204:65-77

Lin KY, Sastri AR, Gong GC, Hsieh CH (2013) Copepod community growth rates in relation to body size, temperature, and food availability in the East China Sea: a test of metabolic theory of ecology. Biogeosciences 10:1877-1892

Lischka S, Hagen W (2005) Life histories of the copepods Pseudocalanus minutus, P. acuspes (Calanoida) and Oithona similis (Cyclopoida) in the Arctic Kongsfjorden (Svalbard). Polar Biol 28:910-921

Liu H, Hopcroft RR (2006a) Growth and development of Neocalanus flemingeri/ plumchrus in the northern Gulf of Alaska: validation of the artificial-cohort method in cold waters. J Plankton Res 28:87-101

Liu H, Hopcroft RR (2006b) Growth and development of Metridia pacifica (Copepoda: Calanoida) in the northern Gulf of Alaska. J Plankton Res 28:769-781

Madsen SD, Nielsen TG, Hansen BW (2008) Annual population development and production by small copepods in Disko Bay, western Greenland. Mar Biol 155:63-77

McKinnon AD (1996) Growth and development in the subtropical copepod Acrocalanus gibber. Limnol Oceanogr 41:1438-1447

McKinnon AD, Duggan S (2003) Summer copepod production in subtropical waters adjacent to Australia's North West Cape. Mar Biol 143:897-907

Peterson WT, Tisels P, Kiørboe T (1991) Copepod egg production, moulting and growth rates, and secondary production, in the Skagerrak in August 1988. J Plankton Res 13:131-154

Postel L, Fock H, Hagen W (2000) Zooplankton methodology manual. Academic, London

Price CA, Weitz JS, Savage VM, Stegen J, Clarke A, Coomes DA, Dodds PS, Etienne RS, Kerkhoff AJ, McCulloh K, Niklas KJ, Olff H, Swenson NG, Chave J (2012) Testing the metabolic theory of ecology. Ecol Lett 15:1465-1474

Runge JA, Roff JC (2000) The measurement of growth and reproductive rates. Academic, London

Svetlichny LS (1983) Calculation of planktonic copepod biomass by means of coefficients of proportionality between volume and linear dimensions of the body. Ekol Morya 15:46-58
Tseng LC, Souissi S, Dahms HU, Chen QC, Hwang JS (2008) Copepod communities related to water masses in the southwest East China Sea. Helgol Mar Res 62:153-165

Turner JT (2004) The importance of small planktonic copepods and their roles in pelagic marine food webs. Zool Stud 43:255-266

Verity PG, Smetacek V (1996) Organism life cycles, predation, and the structure of marine pelagic ecosystems. Mar Ecol Progr Ser 130:277-293

doi:10.1186/1810-522X-52-14

Cite this article as: Lin et al: An alternative kernel-based method for estimating copepod growth rates from multimodal biomass distributions in artificial cohort experiments. Zoological Studies $201352: 14$.

\section{Submit your manuscript to a SpringerOpen ${ }^{\mathcal{D}}$ journal and benefit from:}

- Convenient online submission

- Rigorous peer review

- Immediate publication on acceptance

- Open access: articles freely available online

- High visibility within the field

- Retaining the copyright to your article

Submit your next manuscript at $>$ springeropen.com 